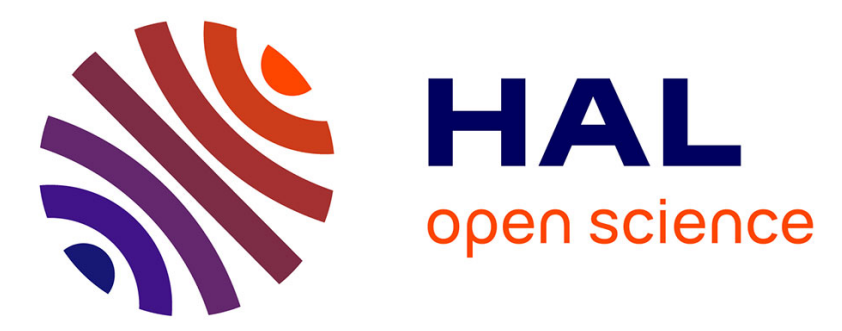

\title{
Set-Membership Position Estimation With GNSS Pseudorange Error Mitigation Using Lane-Boundary Measurements
}

Luís Conde Bento, Philippe Bonnifait, Urbano Nunes

\section{To cite this version:}

Luís Conde Bento, Philippe Bonnifait, Urbano Nunes. Set-Membership Position Estimation With GNSS Pseudorange Error Mitigation Using Lane-Boundary Measurements. IEEE Transactions on Intelligent Transportation Systems, 2019, 20 (1), pp.185-194. 10.1109/TITS.2018.2808542 . hal01739465

\section{HAL Id: hal-01739465 \\ https://hal.science/hal-01739465}

Submitted on 21 Mar 2018

HAL is a multi-disciplinary open access archive for the deposit and dissemination of scientific research documents, whether they are published or not. The documents may come from teaching and research institutions in France or abroad, or from public or private research centers.
L'archive ouverte pluridisciplinaire HAL, est destinée au dépôt et à la diffusion de documents scientifiques de niveau recherche, publiés ou non, émanant des établissements d'enseignement et de recherche français ou étrangers, des laboratoires publics ou privés. 


\title{
Set-Membership Position Estimation With GNSS Pseudorange Error Mitigation Using Lane-Boundary Measurements
}

\author{
Luís Conde Bento ${ }^{(\mathbb{D}}$, Philippe Bonnifait, and Urbano J. Nunes
}

\begin{abstract}
Model-based positioning methods involve nonlinear equations as is the case when using satellite pseudoranges on global navigation satellite systems (GNSSs) and local measurements on road features. As these are nonlinear models, classical estimation methods cannot provide guaranteed position estimation and can converge to local optima, sometimes far away from the global optimum or the true value. Based on interval analysis, set inversion, and constraints propagation on real values provide a framework that guarantees to find the true position with a characterized confidence domain. This paper describes an error bounded set membership algorithm that computes the absolute position of a road vehicle by using raw GNNS pseudoranges, lane boundary measurements, and a $2 \mathrm{D}$ road network map as geometric constraints. The algorithm is based on set inversion using interval analysis, and bounds are set on the measurements by taking into account a chosen risk. The GNSS pseudoranges errors are modeled carefully, and road constraints are formalized to provide additional information in the data fusion process. The proposed algorithm, named lane boundary augmented setmembership GNSS positioning (LB-ASGP), provides a novel and inexpensive approach to improve position estimation performance for road vehicles guaranteeing the enclosure of the computed solution with high confidence. Results from simulations and field experiments show that the LB-ASGP significantly reduces GNSS errors in the direction perpendicular to the lane thanks to the lane boundary measurements.
\end{abstract}

Index Terms-ITS, interval analysis, bounded-error, GNSS and sensor data fusion.

Manuscript received January 27, 2016; revised July 14, 2016, January 31, 2017, March 20, 2017, October 22, 2017, and January 31, 2018; accepted February 4, 2018. This work was supported in part by the Fundação para a Ciência e Tecnologia and FEDER/COMPETE under Project AMSHMI12-RECI/EEIAUT/0181/2012 and Project UID/EEA/00048, and in part by Proj. B-Mobility4People under Grant QREN-MaisCentro SCT-2011-01. The Associate Editor for this paper was A. Amditis. (Corresponding author: Luís Conde Bento.)

L. C. Bento is with the Institute of Systems and Robotics, University of Coimbra, 3004-531 Coimbra, Portugal, and also with the School of Technology and Management, Polytechnic Institute of Leiria, 2411-901 Leiria, Portugal (e-mail: conde@isr.uc.pt).

P. Bonnifait is with the Heudiasyc UMR CNRS 7253, Sorbonne Universités, Université de Technologie de Compiègne, 60200 Compiègne, France (e-mail: philippe.bonnifait@hds.utc.fr).

U. J. Nunes is with the Institute of Systems and Robotics, University of Coimbra, 3004-531 Coimbra, Portugal (e-mail: urbano@isr.uc.pt).

Color versions of one or more of the figures in this paper are available online at http://ieeexplore.ieee.org.

Digital Object Identifier 10.1109/TITS.2018.2808542

\section{INTRODUCTION}

A UTONOMOUS vehicles can lead to more efficient traffic management by relieving traffic congestion, therefore leading to economic and environmental benefits [1], [2]. To implement autonomous driving, position and velocity of the vehicles must be determined with high confidence.

Autonomous navigation widely uses standalone low cost GNSS receivers (single epoch positioning) for vehicle positioning, but these are not accurate enough and are susceptible to provide faulty solutions. To provide integrity it is required to complement GNSS receiver data with other absolute sensor measures. Therefore, the data fusion of multiple and redundant sensory information plays a key role for autonomous vehicle navigation [4].

In [3], a cooperative method to improve position estimation accuracy with integrity is presented. This method named Lane Boundary Cooperative Augmented Set-membership GNSS Positioning ( $L B-C A S G P)$ involves a sensor fusion approach that exploits lane boundary measurements and cooperation between road users through V2X communication to improve both cross-track and along-track vehicle positioning. In this paper we describe an algorithm called Lane Boundary Augmented Set-membership GNSS Positioning ( $L B-A S G P)$, which is a core part of the $L B-C A S G P$ approach [3]. By using local sources of information (such as: GNSS raw measurements, digital maps and lane boundary detection), meaning that it does not require $\mathrm{V} 2 \mathrm{X}$ communication, the $L B-A S G P$ is able to cope with communication shortage events. An improvement to traditional techniques is achieved by guaranteeing, with respect to a chosen integrity risk, that the solution will be found and correctly bounded to the estimated set-membership domain, even with low cost sensors. This is achieved even if the equations are highly non-linear using a bounded-error algorithm. The position improvement achieved by adding laneboundary, can provide sufficient accuracy for driverless cars with additional sensor data like LIDARs [9], [10].

Two algorithms are described in detail and compared:

- $S G P$ - The set membership algorithm proposed in [15];

- $L B-A S G P$ - The proposed algorithm in this paper.

The LB-ASGP algorithm assures the characterization of a bounded solution for vehicle positioning, constrained by geo-referenced lane boundary measurements and GNSS 
pseudoranges to reduce the constellation satellite-specific error. The sensor fusion approach exploits lane boundary measurements to improve cross-track vehicle positioning. When using classical methods for vehicle positioning, the risk arises when trying to bound the obtained estimation error, while in bounded-error models the risk of the solution set not to include the ground truth is only linked to the risk taken when formulating initial assumptions on measurements.

The LB-ASGP inputs are raw GNSS data and lane boundary information acquired thanks to a perception system. The data fusion of different absolute positioning data, in a setmembership algorithm rather than classical nonlinear iterative least squares, is the main contribution of the present study.

Interval algorithms can be easily used to solve a wide spectrum of problems involving uncertainty guaranteeing solution at a defined risk. $L B-A S G P$ is simple to implement thanks to interval algorithms, i.e. the solution set can be rigorously enclosed with a box in a simple way. The proposed method provides also an estimate of the satellite-specific errors along the direction transverse to the road lane boundary. The performance of the $L B-A S G P$ algorithm, in terms of accuracy as well as in terms of integrity are analyzed in simulations and in field experiments.

The remainder of this paper is organized as follows. Section II presents a summary of related work. Section III discusses the components of the GNSS error, GNSS single epoch receiver positioning, lane boundary sensor modelling, set-inversion based on interval analysis and measurement bounds setting. In Section IV, both the $S G P$ and the $L B-A S G P$ set-membership positioning algorithms are formulated and presented. The performance evaluation is presented in Section $\mathrm{V}$ with simulation and experimental results used to test, compare and validate the algorithm. Finally, Section VI summarizes the study and provides concluding remarks.

\section{RELATED WORK}

The use of multiple sensors to improve vehicle positioning accuracy is a common technique applied in Intelligent Transportation Systems (ITS) [5].

The California Partners for Advanced Transit and Highways (PATH) used an on-board sensing system acquiring both the vehicle's state and road reference system based on magnets [6]. However in order to achieve good results the distance between magnets is required to be low, increasing the infrastructure costs.

Both [7] and [8] used cameras to detect lane marking and improve pose estimation. A LIDAR-based lane marker detection has been proposed in [9] to robustly estimate deviations between a digital map and the real world. A localization method where the fusion of a mono-camera, a low-cost GNSS and a map data is presented in [7]. This method uses lateral spatial information from a vision-based lane detection module and longitudinal and lateral spatial information provided by a vision-based traffic sign detection module for high-accuracy positioning. The proposed method achieves centimeter-level localization accuracy and does not need any change on the infrastructure environment. In [8], a lane marking aided vehicle localization uses a lane detection system to retrieve accurate lateral and orientation vehicle information with respect to road lane markings, and combines this information with GNSS estimates and dead-reckoning in order to provide localization information with high availability. A shaping filter of the GNSS errors combining random constant and autoregressive models was implemented. Results show that the filter with this modelling is able to fuse continuous GNSS fixes even if they are affected by large errors.

In [11] and [12], a set of vehicles equipped with GNSS receivers and a lane-boundary sensor, share their information through a V2V network. Using a collaborative navigation algorithm, the satellite-specific GNSS biases experienced by all collaborators is estimated. The error-free differential corrections are more accurate when the vehicle density is high.

The previously mentioned algorithms compute position from GNSS measurements and are based on punctual iterative methods that can be made robust to erroneous measurements. Their main drawback is the risk to fall into a local minimum if the initial guess is too far from the solution, or if erroneous measurements are not properly handled. In [13], a combination of a 3D ray-tracing method, GPS positioning algorithm, particle filter, digital elevation model data, and 2D map data, enabled the detection of NLOS satellites and the improvement of the position accuracy by using an inexpensive GPS receiver. Set-inversion methods guarantee that no solution will be missed inside an arbitrarily big initial box, even if the equations are nonlinear [14].

Safety requires the modeling of any uncertainties associated with the measurement of vehicle positioning. Drevelle and Bonnifait [15], [16] quantified the localization confidence based on interval analysis, set-inversion methods and constraint propagation algorithms. By computing disconnected solution sets, the set-inversion methods can also handle several hypotheses in cases of ambiguous solutions.

\section{THEORETICAL BACKGROUND}

\section{A. GNSS Pseudoranges}

The measured distance from the satellite $s$ to the receiver $r$, is prone to delays from gases ionized by solar radiation $I_{r}^{S}$ (ionospheric delay: up to $50 \mathrm{~m}$ for low elevation satellites) and delays from water vapor $T_{r}^{s}$ (tropospheric delay: from $2.5 \mathrm{~m}$ at the zenith to $15 \mathrm{~m}$ for low satellite elevations). Besides physical phenomena errors, additional system errors include satellite orbit estimation errors $E^{s}$, the clock offset errors $c o_{r}\left(c o_{r}=c \cdot\left(\delta t_{r}-\delta t^{s}\right)\right.$, where $c$ is the speed of light in the vacuum), relativistic errors, diffuse multipath and thermal noise errors $\epsilon_{r}^{s}$.

A pseudorange measurement is given by the following equation [20]:

$$
\begin{aligned}
\rho_{r}^{s}=\sqrt{\left(x^{s}-x_{r}\right)^{2}+\left(y^{s}-y_{r}\right)^{2}+} & \left(z^{s}-z_{r}\right)^{2} \\
+ & +I_{r}^{s}+T_{r}^{s}+E^{s}+\epsilon_{r}^{s}
\end{aligned}
$$

or equivalently:

$$
\rho_{r}^{s}=\left\|\mathbf{x}^{\mathbf{s}}-\mathbf{x}_{\mathbf{r}}\right\|+c o_{r}+v_{r}^{s}
$$

where $v_{r}^{s}$ represent all errors, $\mathbf{x}_{\mathbf{r}}=\left(x_{r}, y_{r}, z_{r}\right)$ and $\mathbf{x}^{\mathbf{s}}=$ $\left(x^{s}, y^{s}, z^{s}\right)$ denote the position vectors of the receiver at the 


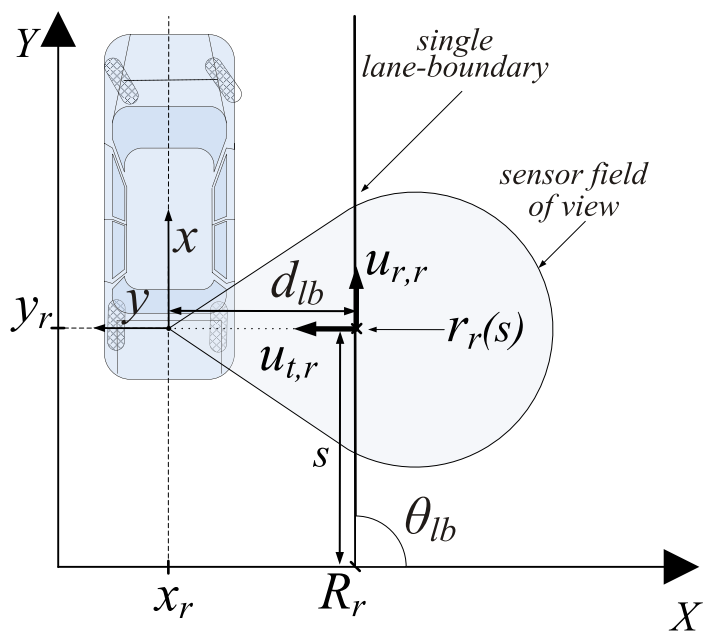

Fig. 1. Geometric layout of lane-boundary distance measurement.

observation epoch (signal reception time) and the satellite at the emission time, respectively. To compute the receiver position $\mathbf{x}_{\mathbf{r}}$ and the receiver clock offset $c o_{r}$, it is required to have at least four satellites in view.

\section{B. Lane Boundary Absolute Measurements}

Road networks can supply additional source of absolute positioning data, provided that the lane boundary is marked on the road and that it is geo-referenced in a Geographic Information System (GIS). Geo-referenced lane boundaries provide means to reduce vehicle positioning error $\left(\hat{\mathbf{x}}_{r}\right.$ and $\left.\hat{c o}_{r}\right)$.

Figure 1 illustrates the geometric layout, considering that the lane-boundary sensor supposed here to be collocated with the GNSS receiver, where $r_{r}(s)$ is the function describing the lane-boundary $2 \mathrm{D}$ coordinates, $s$ is the distance from the reference point $R_{r}$ to the lane-boundary frame $\left\{u_{t, r} ; u_{r, r}\right\}$, $\mathbf{u}_{\mathbf{t}, \mathbf{r}}$ is the transverse unit vector, $d_{l b}$ is the perpendicular distance from the sensor to the lane boundary, and $\theta_{l b}$ is the angle of the lane. Lane-boundary sensors cannot measure location along the lane as they provide only the distance to the lane markings. The transverse component of the distance to the lane boundary $\left(d_{l b}\right)$ can be expressed by:

$$
d_{l b}=-\mathbf{u}_{\mathbf{t}, \mathbf{r}}^{T} \cdot\left(R_{r}-\mathbf{x}_{\mathbf{r}}\right)+\epsilon_{r}^{l b}
$$

where $\epsilon_{r}^{l b}$ models the sensor measurement errors.

\section{Set Inversion}

Set-membership methods [22] solve the inversion problem in a different way than Iterative least squares [20], which are commonly used for vehicle positioning. The Cartesian product of $n$ intervals is an interval vector here denoted by $[\mathbf{x}]=[\overline{\mathbf{x}}, \underline{\mathbf{x}}]$. An interval vector is a subset of $\mathbb{R}^{n}$, also designated as box. The interval function $[\mathbf{f}]: \mathbb{I R}^{n} \rightarrow \mathbb{I R}^{m}$ is an inclusion function for $\mathbf{f}$ if

$$
\forall[\mathbf{x}] \in \mathbb{I R}^{n}, \mathbf{f}([\mathbf{x}]) \subset[\mathbf{f}]([\mathbf{x}])
$$

where $\mathbf{f}$ defines a mapping $\mathbb{R}^{n} \rightarrow \mathbb{R}^{m}, \mathbb{I} \mathbb{R}$ is the set of real intervals and $\mathbb{I R}^{n}$ is the set of $\mathrm{n}$-dimensional boxes. A box $[\mathbf{x}]$
TABLE I

\begin{tabular}{|c|c|c|}
\hline Test Result & Condition & Action \\
\hline Feasible boxes & $\begin{array}{c}{[\mathbf{x}] \text { is feasible if }} \\
{[\mathbf{f}]([\mathbf{x}]) \subset \mathbb{Y}}\end{array}$ & $\begin{array}{l}\text { boxes are added to } \\
\text { the inner sub-paving } \\
\underline{X}\end{array}$ \\
\hline Unfeasible boxes & $\begin{array}{l}{[\mathbf{x}] \text { is unfeasible if }} \\
{[\mathbf{f}]([\mathbf{x}]) \cap \mathbb{Y}=\emptyset}\end{array}$ & boxes are discarded \\
\hline $\begin{array}{l}\text { Indeterminate box } \\
\quad \text { width } \geq \varepsilon\end{array}$ & $\begin{array}{l}{[\mathbf{x}] \text { is }} \\
\text { indeterminate if } \\
{[\mathbf{f}]([\mathbf{x}]) \text { intersects }}\end{array}$ & $\begin{array}{l}\text { bisected into two } \\
\text { sub-boxes } \\
\text { rescheduled to be } \\
\text { retested }\end{array}$ \\
\hline $\begin{array}{c}\text { Indeterminate box } \\
\text { width }<\varepsilon\end{array}$ & $\begin{array}{l}\text { but is not } \\
\text { included in } \mathbb{Y}\end{array}$ & $\begin{array}{l}\text { boxes are added to } \\
\text { the sub-paving } \Delta \mathbb{X}\end{array}$ \\
\hline
\end{tabular}

Test Boxes For Set Membership

can be refined by using a sub-paving. A sub-paving can be defined as the union of non-overlapping sub-boxes, excluding all zero-sized boxes. Bracketed between an inner sub-paving $\underline{\mathbb{X}}$ and an outer sub-paving $\overline{\mathbb{X}}$, lies the compact set $\mathbb{X}$, where $\underline{\mathbb{X}} \subset \mathbb{X} \subset \overline{\mathbb{X}}$. Using the Set Inversion Via Interval Analysis (SIVIA) algorithm [23], an outer approximation $\overline{\mathbb{X}}$ to the solution set $\mathbb{X}$ can be computed.

The procedure to compute the outer sub-paving $\overline{\mathbb{X}}$ of the set, that fulfills positioning constraints, starts by considering a sufficiently large superset $\mathbb{X}_{0}$ to contain the solution set. A test on each box $[\mathbf{x}]$ is performed using the set inversion algorithm, resulting in one of three outputs and consequent actions (see Table I). Following the box feasibility test, one can compute the outer sub-paving $\overline{\mathbb{X}}=\underline{\mathbb{X}}+\Delta \mathbb{X}$.

The inverse evaluation problem is characterized by [18]:

$$
\mathbb{X}=\mathbf{f}^{-1}(\mathbb{Y})=\left\{\mathbf{x} \in \mathbb{R}^{n} \mid \exists \mathbf{y} \in \mathbb{Y}, \mathbf{f}(\mathbf{x})=\mathbf{y}\right\}
$$

where $\mathbb{Y}$ is a known interval vector of $m$ measurements. The set inversion problem consists in determining the set $\mathbb{X}$, such that $\mathbf{f}(\mathbb{X}) \subset \mathbb{Y}$, where $\mathbb{Y} \subset \mathbb{R}^{m}$.

\section{Measurement Bounds Setting}

Based on set theory, bounded-error methods can compute a solution set not necessarily of minimum size, but guaranteed to fulfill the integrity risk requirement [3], [17].

The error measurement model to set the error bounds on each pseudorange measurement $\rho_{r}^{s}$ is assumed to follow a centered Gaussian distribution. The measurement error bound $\alpha$ is chosen while taking into account the maximum risk $r$ of each measurement interval to not contain the actual value. Given a centered Gaussian distribution with a variance $\sigma_{p r}^{2}$ and a cumulative distribution function of the standard normal distribution $\Phi$, the measurement error bounds $\alpha$ are given by:

$$
\left\{\begin{array}{l}
{\left[\rho_{r}^{s}\right]=\left[\rho_{r}^{s}-\alpha \sigma_{p r}, \rho_{r}^{s}+\alpha \sigma_{p r}\right]} \\
\alpha=-\Phi^{-1}\left(\frac{r}{2}\right)
\end{array}\right.
$$

\section{Position Estimation Algorithms}

Computing a set where a vehicle is located is achieved using bounded errors measurements. Set-inversion methods [22] are very well adapted for this type of problems by a twofold reason: 1) It is guaranteed that there is no loss of solutions; 


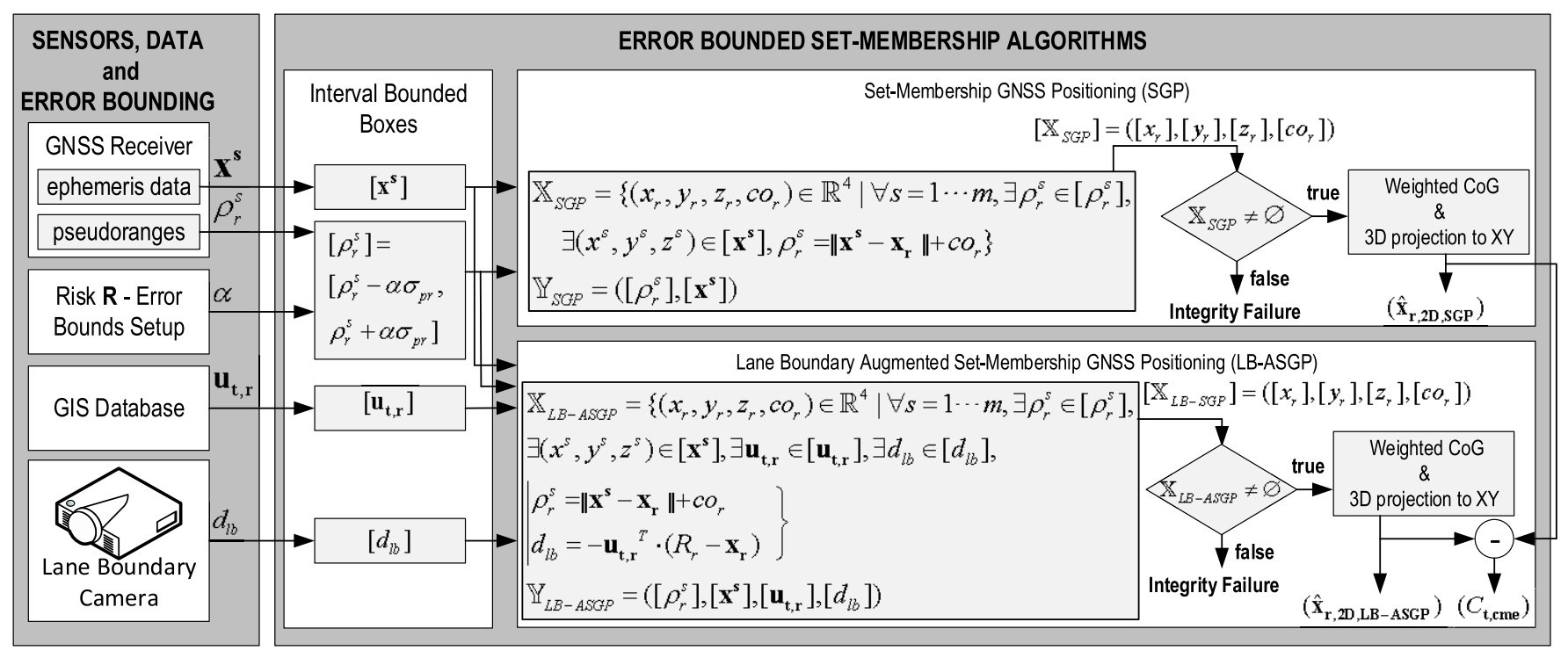

Fig. 2. Dataflow and architectures of SGP and LB-ASGP algorithms.

2) No risk is added in the computation of a compact set, i.e. the risk that the pseudoranges true value is not contained in the measurements interval will determine the risk that the true receiver position is not on the position set.

\section{A. Set Membership GNSS Positioning}

In order to compute a location set, where the vehicle is guaranteed to be at known risk, the Set membership GNSS Positioning ( $S G P$ ) method [11] requires that its inputs embody the uncertainty in the form of interval vectors. Therefore both pseudorange measurements and the satellite positions are represented as boxes, whose bounds are determined given a chosen risk, $\left[\mathbf{x}^{\mathbf{s}}\right]=\left(\left[x^{s}\right],\left[y^{s}\right],\left[z^{s}\right]\right)$ and $\left[\rho_{r}^{s}\right]$ represent the interval vectors for the satellite positions and pseudorange measurements respectively [15].

Given the satellites' positions $\left[\mathbf{x}^{\mathbf{s}}\right]=\left(\left[x^{s}\right],\left[y^{s}\right],\left[z^{s}\right]\right)$ and the $m$ available pseudorange measurements, it is possible to compute the set $\mathbb{X}$ compliant with the inputs stated above:

$$
\begin{aligned}
\mathbb{X}_{S G P}= & \left\{\left(x_{r}, y_{r}, z_{r}, c o_{r}\right) \in \mathbb{R}^{4} \mid \forall s=1 \cdots m, \exists \rho_{r}^{s} \in\left[\rho_{r}^{s}\right],\right. \\
& \left.\exists\left(x^{s}, y^{s}, z^{s}\right) \in\left[\mathbf{x}^{\mathbf{s}}\right], \rho_{r}^{s}=\left\|\mathbf{x}^{\mathbf{s}}-\mathbf{x}_{\mathbf{r}}\right\|+c o_{r}\right\} \\
\mathbb{Y}_{S G P}= & \left(\left[\rho_{r}^{s}\right],\left[\mathbf{x}^{\mathbf{s}}\right]\right)
\end{aligned}
$$

$\mathbb{X}$ is subset of $\mathbb{R}^{4}$, composed by a finite list of boxes $[X][22]$, where $\# \mathbb{X}$ is the number of boxes belonging to the subset. The set $\mathbb{X}$ is a $4 \mathrm{D}$ set, composed of $4 \mathrm{D}$ boxes with their axis along $x_{r}, y_{r}, z_{r}$ and $c o_{r}$. A 2D punctual estimate is computed by projecting to the $X Y$ plane the center of all boxes weighted by two parameters:

- The volume of each box: inner sub-paving boxes having not the same size contribute differently, therefore large boxes have to contribute more than small ones;

- The distance from a weighted average of $\hat{c o_{r}}$ : Boxes with a large time uncertainty $\mathrm{co}_{r}$ are less accurate for positioning. Therefore, the weight should be higher for boxes closer to the $\hat{\mathrm{co}_{r}}$ estimate.
The $\hat{c o}$ estimate is obtained by:

$$
\begin{aligned}
& \hat{c o}{ }_{r}=\frac{\sum_{k=1}^{n}\left(\frac{\overline{X_{k}(4)}+\underline{X_{k}(4)}}{2} \cdot\left(\overline{X_{k}(4)}-\underline{\left.X_{k}(4)\right)}\right)\right.}{X_{n o r m}(4)} \mid \\
& \forall\left[X_{k}(4)\right] \in \mathbb{X} \wedge k=\# \mathbb{X}
\end{aligned}
$$

The $3 \mathrm{D}$ position estimate $\hat{\mathbf{x}}_{r}=\left(\hat{x}_{r}, \hat{y}_{r}, \hat{z}_{r}\right)$ is:

$$
\begin{aligned}
& \hat{\mathbf{x}}_{r}=\frac{\sum_{k=1}^{n}\left(\frac{\overline{X_{k}(i)}+\underline{X_{k}(i)}}{2} \cdot\left(\overline{X_{k}(i)}-\underline{X_{k}(i)}\right) \cdot C_{w f}(k)\right)}{X_{\text {norm }}(i)} \\
& \forall\left[X_{k}(i)\right] \in \mathbb{X} \wedge k=\# \mathbb{X} \wedge i=1, \cdots, 3
\end{aligned}
$$

Where $X_{n o r m}$ is the normalization term and $C_{w f}$ is the weighting factor, both are computed has described in [3].

The $\hat{\mathbf{x}}_{r}$ estimate is the center of gravity of the sub-paving, weighted by the volume as well as the distance from a weighted average of $\hat{c o} r$ of each sub-paving. The projection of the $\hat{\mathbf{x}}_{r}$ to the $X Y$ plane provides the 2D position estimate $\hat{\mathbf{x}}_{r, 2 D, S G P \text {. }}$

\section{B. Lane Boundary Augmented Set Membership GNSS Positioning (LB-ASGP)}

Constraints provided by lane-boundary can further improve the performance of the set-membership GNSS location zone determination (see Fig. 2).

An arbitrary number of sensors can be fused by means of set-membership methodologies, given that both the model and the measurement errors are bounded [19]. Under the assumption of correct bounds on models and measurements, set-membership algorithms provide guaranteed results up to the chosen integrity risk. The system composed of a laneboundary sensor and a GIS provides an additional constraint to a box $[\mathbf{x}]$, in the direction transverse to the road.

To represent the uncertainties of the information stored in the GIS, the transverse $\mathbf{u}_{\mathbf{t}, \mathbf{r}}$ vector is represented by 
a box $\left[\mathbf{u}_{\mathbf{t}, \mathbf{r}}\right]$. Similarly, the orthogonal distance from the vehicle $x$-axis to the lane-boundary $d_{l b}$ is inaccurate and therefore is also represented by a box $\left[d_{l b}\right]$.

With the additional lane-boundary sensor measurements in the SIVIA algorithm, the search space is further reduced:

$$
\begin{aligned}
\mathbb{X}_{L B-A S G P}= & \left\{\left(x_{r}, y_{r}, z_{r}, c o_{r}\right) \in \mathbb{R}^{4} \mid \forall s=1 \cdots m, \exists \rho_{r}^{s} \in\left[\rho_{r}^{s}\right],\right. \\
& \exists\left(x^{s}, y^{s}, z^{s}\right) \in\left[\mathbf{x}^{\mathbf{s}}\right], \exists \mathbf{u}_{\mathbf{t}, \mathbf{r}} \in\left[\mathbf{u}_{\mathbf{t}, \mathbf{r}}\right], \exists d_{l b} \in\left[d_{l b}\right], \\
& \left.\begin{array}{l}
\rho_{r}^{s}=\left\|\mathbf{x}^{\mathbf{s}}-\mathbf{x}_{\mathbf{r}}\right\|+c o_{r} \\
d_{l b}=-\mathbf{u}_{\mathbf{t}, \mathbf{r}}^{T} \cdot\left(R_{r}-\mathbf{x}_{\mathbf{r}}\right)
\end{array}\right\} \\
\mathbb{Y}_{L B-A S G P}= & \left(\left[\rho_{r}^{s}\right],\left[\mathbf{x}^{\mathbf{s}}\right],\left[\mathbf{u}_{\mathbf{t}, \mathbf{r}}\right],\left[d_{l b}\right]\right)
\end{aligned}
$$

In order to have an outer approximation $\overline{\mathbb{X}}$ of the localization set $\mathbb{X}$ by using SIVIA, it is necessary to start with a set $\mathbb{X}_{0}$ where the solution set is known to belong. The LB-ASGP can be divided into three stages (see Algorithm 1):

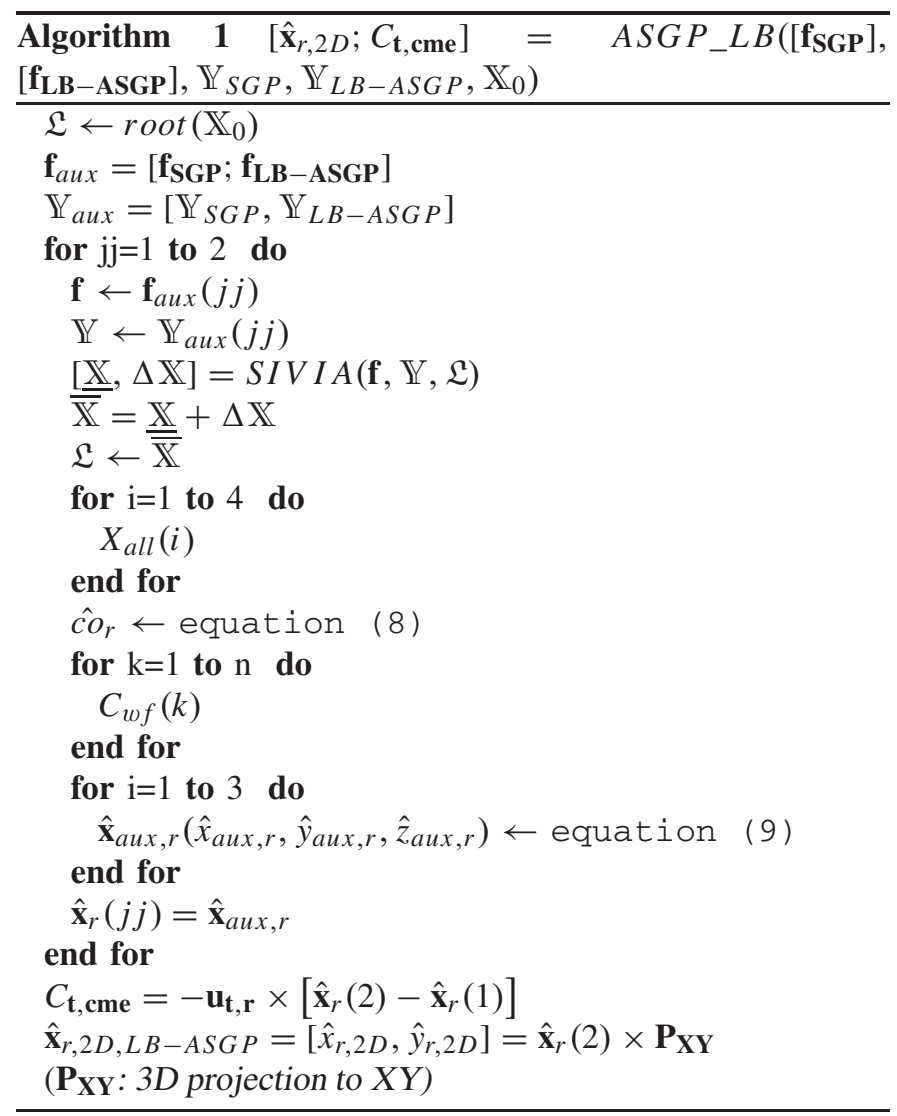

- Compute the set $\mathbb{X}_{S G P} \Rightarrow$ starting with a large superset $\mathbb{X}_{0}$ and given the measurements $\left[\rho_{r}^{s}\right]$ and the satellite position [ $\mathbf{x}^{\mathbf{s}}$ ], compute the set $\mathbb{X}_{S G P}$ using function $\mathbf{f}_{\mathbf{S G P}}$ given by: $\mathbf{f}_{\mathbf{S G P}}=\left\{\rho_{r}^{s}=\left\|\mathbf{x}^{\mathbf{s}}-\mathbf{x}_{\mathbf{r}}\right\|+c o_{r}\right.$;

- Compute the set $\mathbb{X}_{L B-A S G P} \Rightarrow$ starting with $\mathbb{X}_{S G P}$ and given the measurements $\left[\rho_{r}^{s}\right]$, the satellite position $\left[\mathbf{x}^{\mathbf{s}}\right]$, the transverse vector $\left[\mathbf{u}_{\mathbf{t}, \mathbf{r}}\right]$ and the lane boundary $\left[d_{l b}\right]$, compute the set $\mathbb{X}_{L B-A S G P}$ using function $\mathbf{f}_{\mathbf{L B}-\mathbf{A S G P}}$ given by: $\mathbf{f}_{\mathbf{L B}-\mathbf{A S G P}}=\left\{\begin{array}{l}\rho_{r}^{s}=\left\|\mathbf{x}^{\mathbf{S}}-\mathbf{x}_{\mathbf{r}}\right\|+c o_{r} \\ d_{l b}=-\mathbf{u}_{\mathbf{t}, \mathbf{r}}{ }^{T} \cdot\left(R_{r}-\mathbf{x}_{\mathbf{r}}\right)\end{array} ;\right.$

- Compute the 2D estimates $\hat{\mathbf{x}}_{r, 2 D, S G P}$ and $\hat{\mathbf{x}}_{r, 2 D, L B-A S G P} \Rightarrow$ The $3 \mathrm{D}$ estimates $\hat{\mathbf{x}}_{r, 3 D, S G P}$ and $\hat{\mathbf{x}}_{r, 3 D, L B-A S G P}$ are computed, using sets $\mathbb{X}_{S G P}$ and
$\mathbb{X}_{L B-A S G P}$ respectively (equations 8 and 9). Finally, 2D position estimates $\hat{\mathbf{x}}_{r, 2 D, S G P}$ and $\hat{\mathbf{x}}_{r, 2 D, L B-A S G P}$ are computed.

Algorithm 1 additionally provides an estimate of the satellite-specific errors $C_{t, c m e}$, along the direction transverse to the lane boundary. This component reflects the errors which causes the receiver position estimate to suffer from an offset in the direction orthogonal to the lane boundary marking. The $C_{t, c m e}$ is obtained by taking into account only the road transverse component that has shifted the estimated position obtained using the $S G P$ algorithm to the estimated position obtained using $L B-A S G P: C_{\mathbf{t}, \mathbf{c m e}}=\mathbf{u}_{\mathbf{t}, \mathbf{r}} \times$ $\left[\hat{\mathbf{x}}_{r, L B-A S G P}-\hat{\mathbf{x}}_{r, S G P}\right]$ (see Fig. 2).

\section{RESULTS}

Simulations and field experiments were conducted, to analyze the performance of the proposed LB-ASGP algorithm in comparison with SGP. In simulation, the performance of the set-membership based algorithms is compared to standard iterative least squares positioning (IGP) and augmented iterative least squares algorithms (LB-AIGP).

\section{A. Simulation Setup}

A Matlab-based open-source simulator ISR-TRAFSIM 4.0 has been used as the simulation environment (available at: http://home.isr.uc.pt/ conde/isr-trafsim/). The simulated scenario consists of a urban road network presented in Fig. 6(a), where six vehicles travel. In Fig. 6(a), the starting vehicle positions and their headings are depicted: each vehicle is marked with a pair of coordinate axes indicating local vehicle $\mathrm{x}$-axis and $\mathrm{y}$-axis which represent along-track and cross-track directions respectively. To emulate the GNSS constellation and the signals propagation, the GPSoft [21] software was used. GPSoft enables emulation of Glonass, Galileo, GEOs, GPS and GPS Modernization (C/A-code on L1, L2 and L5) as well as dual-frequency P-code measurements. The emulation of C/A and P-code pseudorange and integrated Doppler with user definable civil and military carrier frequencies is available including characteristics such as: thermal noise, ionospheric delay, tropospheric delay and diffuse multipath [21]. The errors are emulated such that the proper temporal and spatial correlation effects are observed in the measurements, their characteristics are the following:

- Ionospheric - the average injected error is 4 meters, emulated through a half cosine function of the local time scaled by the satellite elevation factor;

- Tropospheric - the injected error ranges from 3 meters for a satellite at zenith to 25 meters for a satellite at 5 degrees elevation;

- Diffuse multipath - standard deviation of pseudorange diffuse multipath errors at zero-elevation is 1.6 meters. Each satellite/receiver pair has its own uncorrelated diffuse multipath error.

- Thermal noise - 1 meter of standard deviation.

The C/A-L1 code was used to emulate the satellite-receiver signal travelling from the satellite $s$ to the receiver $r$.

The bounds on pseudoranges are computed using $\left[\rho_{r}^{s}\right]=$ $\left[\rho_{r}^{s}-\alpha \sigma_{p r}, \rho_{r}^{s}+\alpha \sigma_{p r}\right]$ (see equation 6). Table II presents the 

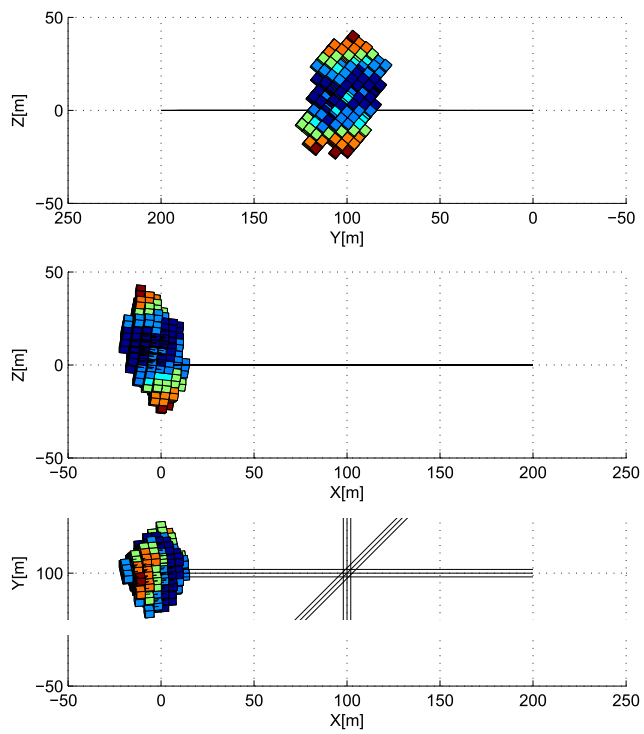

(a)
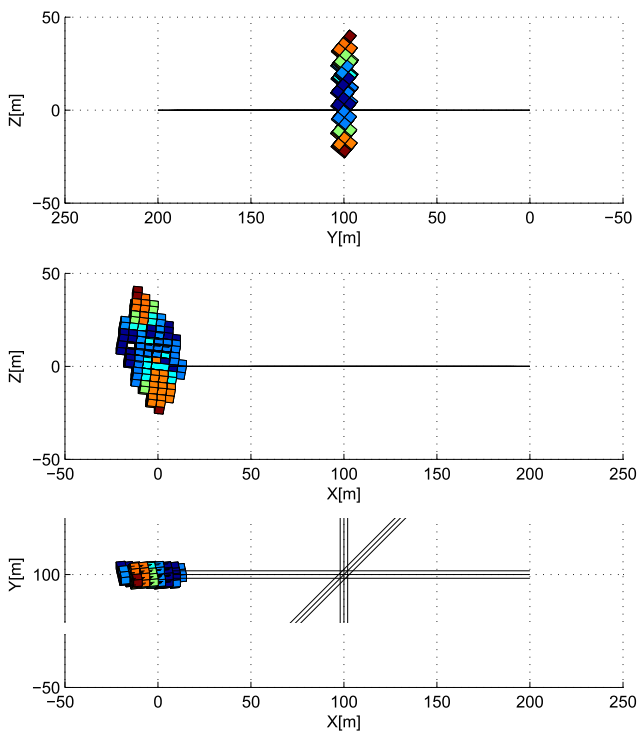

(b)

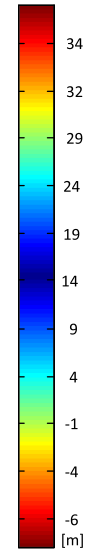

Fig. 3. Simulation - Vehicle 1 - 2D1/2 set-membership solution (a) and 2D1/2 lane-boundary constrained set-membership solution (b): (top) 3D projection onto the YZ plane (lateral distribution); (middle) 3D projection onto the XZ plane (longitudinal distribution); (bottom) 3D projection onto the XY plane (top view distribution). The clock offset $c o_{r}$ follows the gradient of (c).

TABLE II

PSEUDORANGE INTERVALS RISK $r$ AND $\alpha$ ERROR BOUNDS AS A FunCtion OF THE NUMBeR $m$ OF MEASUREMENTS

\begin{tabular}{c|cccc}
\hline \hline$m$ & 4 & 5 & 6 & 7 \\
\hline$r$ & $0.25 \cdot 10^{-4}$ & $0.20 \cdot 10^{-4}$ & $0.17 \cdot 10^{-4}$ & $0.14 \cdot 10^{-4}$ \\
$\alpha$ & 4.21 & 4.26 & 4.31 & 4.34 \\
\hline \hline$m$ & 8 & 9 & 10 & 11 \\
\hline$r$ & $0.13 \cdot 10^{-4}$ & $0.11 \cdot 10^{-4}$ & $0.10 \cdot 10^{-4}$ & $0.09 \cdot 10^{-4}$ \\
$\alpha$ & 4.37 & 4.39 & 4.42 & 4.44
\end{tabular}

pseudorange intervals risk $r$ and the error bounds $\alpha$, given the global risk $\mathbf{R}=10^{-4}$ and the number $m$ of measurements. The lane-boundary sensor error is characterized by a standard deviation of $\sigma_{l b}=0.25 \mathrm{~m}$.

\section{B. Experimental Setup}

The automotive setup consisted of two standard road vehicles. Two low cost GNSS receivers with raw-data capabilities were mounted on each vehicle, spaced 2 meters longitudinally. Therefore, four sources of GNSS data were available, enabling the test of the algorithm has if it were four vehicles. Additionally, each vehicle was also equipped with a centimeter grade GNSS receiver used as ground-truth. The ground-truth receiver was setup in RTK-GPS mode using a network of permanent reference GNSS stations. The test site cover $0.4 \mathrm{Km}^{2}$ and it has $10 \mathrm{Km}$ of roads, there are trees and buildings spreaded around the site, creating disturbances on the received signals. The following equipment was used in the experimental tests:

- Low cost vehicle GNSS receivers - ublox LEA-6T embedded GPS-receiver;

- High cost ground-truth GNSS receivers - high performance RTK-GPS system TOPCON HiperPro
- RTK network - military network SERVIR capable of providing raw-data observations and corrections for realtime RTK or post-processed PPK

Lane camera measurements were emulated using RTK-GPS with a $25-\mathrm{cm}$ additional white Gaussian noise, modelling both camera and GIS errors [24].

\section{Set-Membership GNSS Positioning (SGP)}

When using set-membership GNSS methods, an important focus is on the characterization of domains which contain the solution, rather than on the search of punctual results which might be misleading. Figure 3 shows the simulation results. The initial searching volume was set to $27 \times 10^{6}\left[\mathrm{~m}^{3}\right]$ which is an arbitrarily high value with little impact on the processing time.

The column (a) subplots of Fig. 3 present the enveloping box for the position of vehicle 1 , located at $\left(x_{r 1}, y_{r 1}, z_{r 1}\right)=$ $(0,100,0)[m]$, when the constraints provided by the satellites constellation are used to reduce the search space of the initial box. If a box does not belong to the solution set, it is not explored anymore and discarded. The wide domains are therefore reduced to a small enclosing set of boxes. The setmembership GNSS positioning location set $\mathbb{X}$, presented on the column (a) subplots of Fig. 3, is made of 407 boxes.

The satellite constellation setup produced a very small bias towards the positive direction of the local coordinates $Y$-axis, i.e. a bias towards the left side of vehicle 1. This bias can be seen easily in the top subplot of Fig. 3(a) where the $3 \mathrm{D}$ projection onto the $\mathrm{YZ}$ plane (lateral distribution) of the set-membership GNSS positioning location set $\mathbb{X}$ is presented. From the bottom subplot of Fig. 3(a), it is possible to observe a small bias towards negative direction of the local coordinates $X$-axis, i.e. the satellite constellation produces a very small bias 
TABLE III

Average of the Absolute Mean Lateral Error

\begin{tabular}{cl|cc}
\hline \hline$\sum_{i=1}^{N} \frac{\| \text { mean lat. error } \|}{N}$ & \multicolumn{2}{c}{ vehicles } \\
\hline \multirow{5}{*}{ Simulation } & SGP [m] & {$[1,3,5,6]$} & {$[1,4]$} \\
& LB-ASGP [m] & 0.685 & 1.92 \\
& IGP [m] & 0.069 & 0.032 \\
& LB-AIGP [m] & 0.023 & 1.75 \\
& & \multicolumn{2}{c}{$[1,2,3,4]$} \\
\hline \multirow{5}{*}{ Real Exp. } & SGP [m] & 0.693 \\
& LB-ASGP [m] & \multicolumn{2}{c}{0.048}
\end{tabular}

TABLE IV

Lateral ERror ANALYSis For All Vehicles

\begin{tabular}{ll|ccc}
\hline \hline \multicolumn{2}{c}{ Lateral error } & mean & $\sigma$ & $3 \sigma$ \\
\hline \multirow{2}{*}{ Simulation } & SGP [m] & 0.068 & 1.437 & 3.2 \\
& LB-ASGP [m] & -0.001 & 0.269 & 0.8 \\
& IGP [m] & 0.057 & 1.339 & 3.0 \\
& LB-AIGP [m] & 0.008 & 0.247 & 0.7 \\
\hline \multirow{2}{*}{ Real Exp. } & SGP [m] & 0.551 & 3.713 & 13 \\
& LB-ASGP [m] & 0.036 & 0.478 & 2.5
\end{tabular}

towards the rear side of vehicle 1 . This bias is more visible on the middle subplot of Fig. 3(a) where the 3D projection onto the XZ plane (longitudinal distribution) of the set-membership GNSS positioning location set $\mathbb{X}$ is presented. One can also observe from the top and middle subplots of Fig. 3 a positive bias along the local coordinates $Z$-axis.

When using the set-membership GNSS positioning location set $\mathbb{X}$, the position estimate $\left[x_{r}, y_{r}\right]$ is determined by projecting the solution of the center of gravity (equations 8 and 9). The clock offset $c o_{r}$ distribution for each paving is depicted in the 2D 1/2 subplot of Fig. 3, where the clock offset $c o_{r}$ follows the gradient of Fig. 3(c).

The simulated vehicles $1,3,5$ and 6 travel in a road lane parallel to the local coordinates $X$-axis, while vehicles 2 and 4 travel in a road lane parallel to the local coordinates $Y$-axis, as shown in Fig. 6(a). The absolute mean lateral error value along the local coordinates $X$-axis is higher than that along the $Y$-axis (see Table III). Therefore, this satellite constellation configuration generates an error with the major axis along the negative direction of the local coordinates $X$-axis.

From the simulation results shown in Fig. 6(a) and Table IV, one can observe that the algorithm has a very small mean lateral error and a high standard deviation $\sigma$. This error profile is due to the fact that vehicles traveling in different directions have opposite error signals. The cumulative $3 \sigma$ boundary of the lateral positioning error for $S G P$ algorithm is very high meaning that the positioning method is rather inaccurate most of the time.

Figures 4 and 5 presents the results achieved in field experiments, where the dashed line with ' ${ }^{*}$ ' markers and the solid line with ' $\square$ ' markers, represent the position errors when using algorithm $(S G P)$ and $(L B-A S G P)$ respectively.

Figure 4 presents the results of the lateral normalized positioning error, while Fig. 5 displays normalized positioning lateral (top), longitudinal (center) and 2D Euclidean (bottom) error distribution, as well as its cumulative distribution functions. From the results depicted in Fig. 4, it is not possible
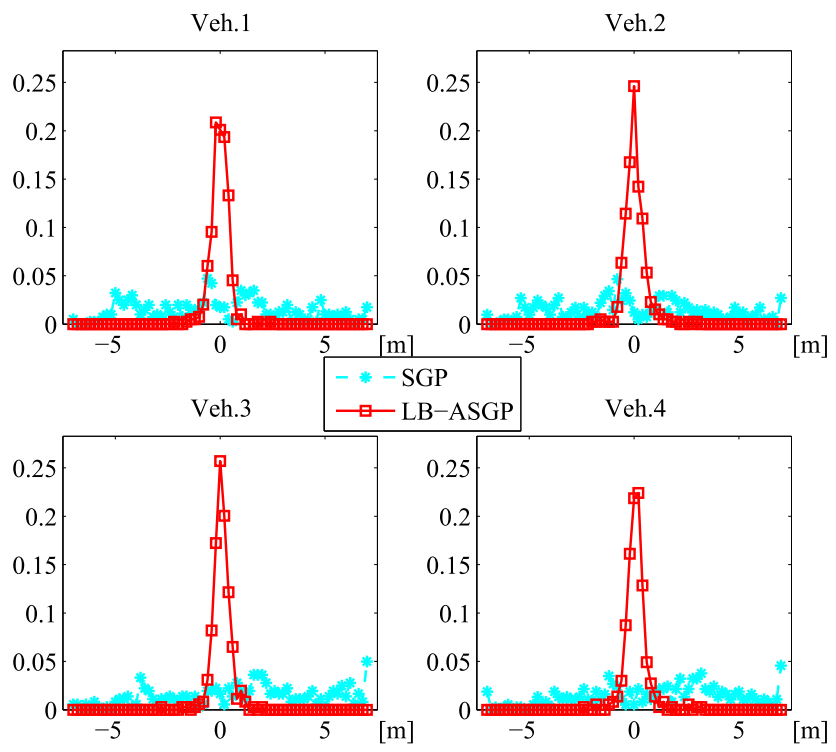

Fig. 4. Field experiments - Lateral normalized positioning error distribution, for all trajectory path of vehicles 1 to 4 , using $S G P$ and $L B-A S G P$ estimation.
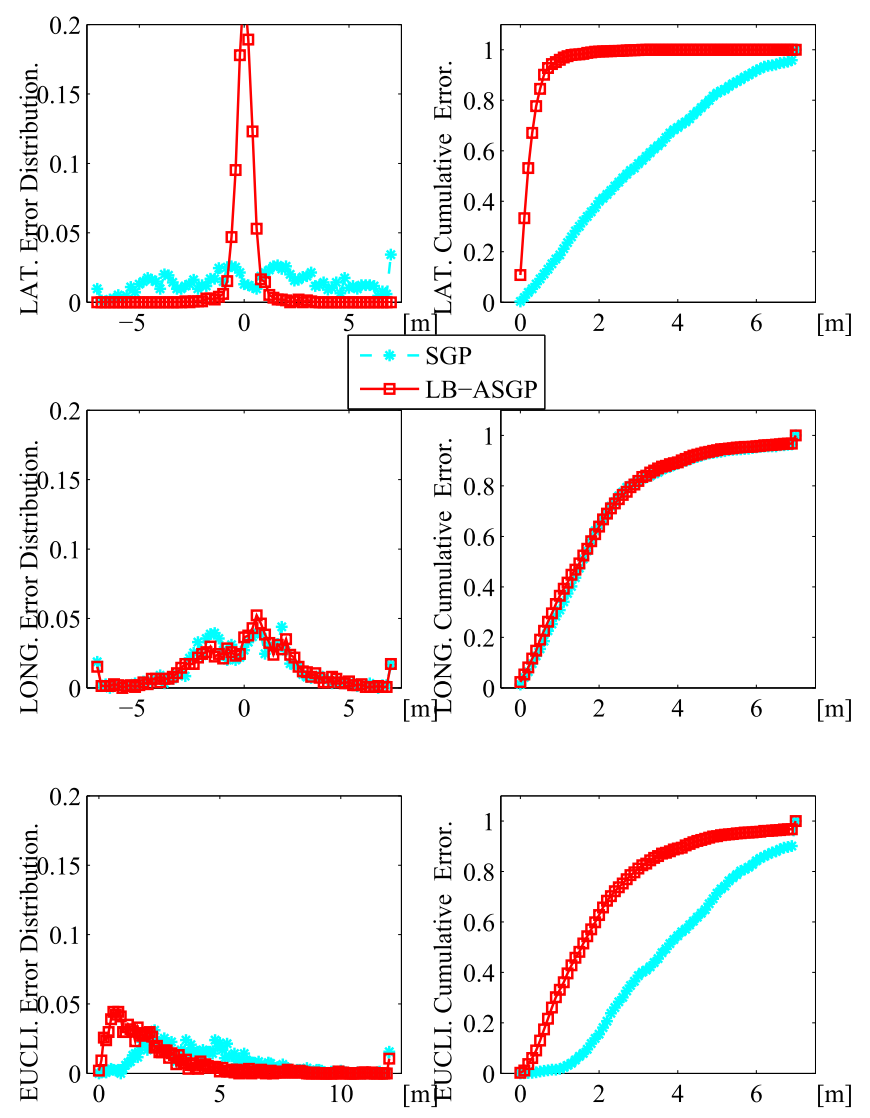

Fig. 5. Field experiments - Vehicles positioning errors, lateral (top), longitudinal (center) and 2D Euclidean (bottom), using SGP and LB-ASGP estimation: (a) normalized positioning errors distribution. (b) cumulative distribution functions.

to observe a predominant axis error, since the trajectories followed by all vehicles have a closed loop shape. The absolute mean lateral error of (SGP) for all the vehicles is moderate, see Table III. The field experimental results, depicted in Fig. 5 and Table IV, confirm the observations made in simulation. 


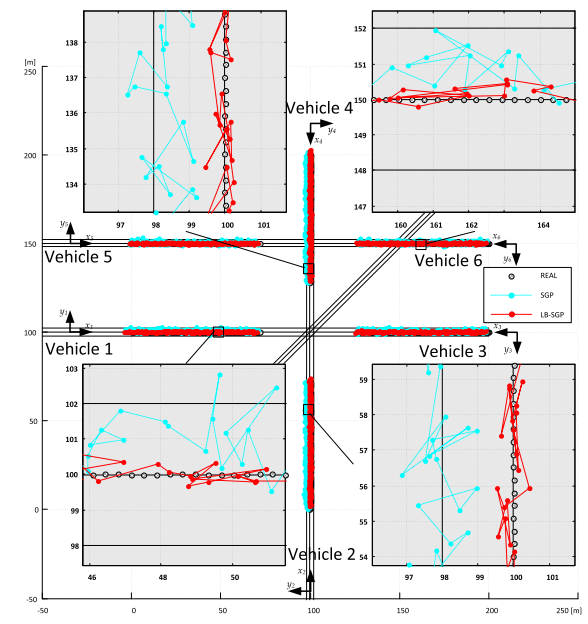

(a)

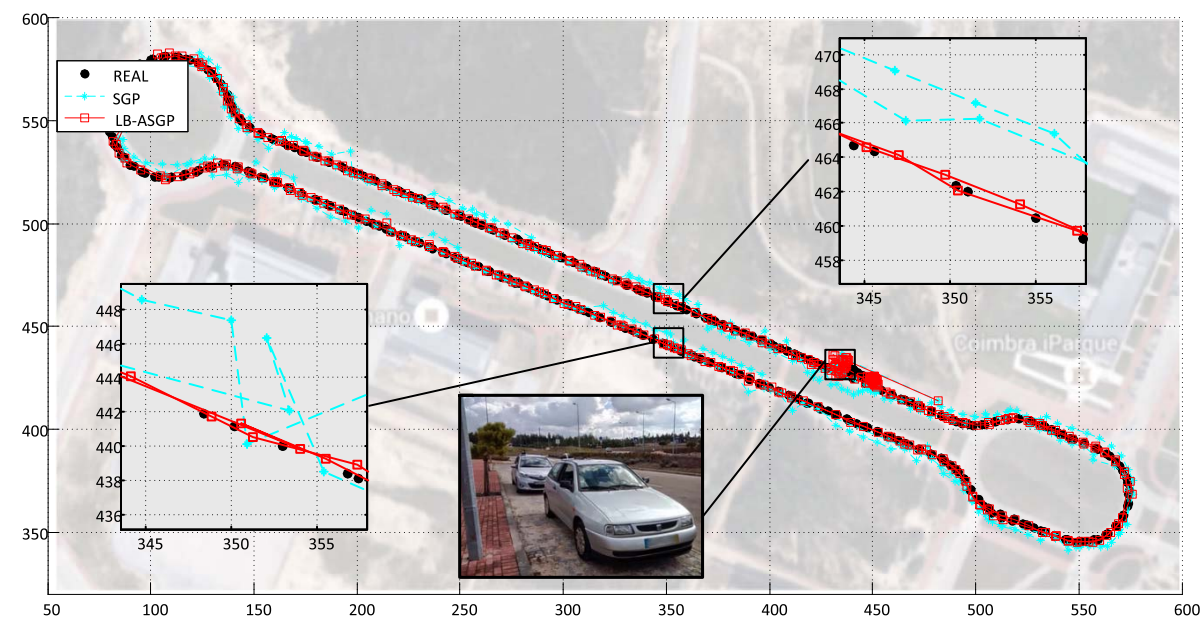

(b)

Fig. 6. Trajectory and zoomed trajectory followed by each vehicle: (a) Simulation - vehicle 1 trajectory in the bottom-left zoom subplot; vehicle 2 trajectory in the bottom-right zoom subplot; vehicle 4 trajectory in the top-left zoom subplot and vehicle 6 trajectory in the top-right zoom. (b) Field experiments

The lateral errors are quite large and highly spread for the $S G P$ algorithm. The cumulative distribution $3 \sigma$ of the 2D Euclidean error is also very high. The mean, standard deviation, maximum and minimum errors in positioning are similar for both $I G P$ and $S G P$ algorithms, being a slightly higher for the $S G P$ algorithm.

\section{Set-Membership Augmented GNSS Positioning (LB-ASGP)}

The results presented in this section make use of the laneboundary measurements. Column (b) subplot of Fig. 3 presents the enveloping box for the position of vehicle 1, located at $\left(x_{r 1}, y_{r 1}, z_{r 1}\right)=(0,100,0)[m]$, when the constraints provided by GIS and lane-boundary sensor are used to reduce to constraint the initial box. The set-membership GNSS positioning location set $\mathbb{X}$ is reduced after a characterization of every zone compatible with the lane-boundary measurements. The resulting lane-boundary set is composed by 137 boxes.

By analyzing the column (b) subplots of Fig. 3, it is possible to observe that the $3 \mathrm{D}$ projection onto the $\mathrm{YZ}$ plane (lateral distribution) of the set-membership GNSS positioning location set $\mathbb{X}$ is significantly reduced. The impact of the positive bias along local coordinates $Y$-axis that this satellite constellation configuration generates on vehicle 1 is also reduced as a result. It is possible to observe that the $3 \mathrm{D}$ projection onto the $\mathrm{XZ}$ plane (longitudinal distribution) of the set-membership GNSS positioning location set $\mathbb{X}$, maintains its shape, i.e. the impact of the predominant negative error along local coordinates $X$-axis generated by this satellite constellation configuration on vehicle 1 is not reduced.

After having applied the lane-boundary constrain on the estimated set $\mathbb{X}$, a new position estimate is performed. The $L B$-ASGP algorithm has considerably less mean absolute lateral error than $S G P$, with an error reduction of at least $90 \%$. From top subplots of Fig. 5 and Table IV one can observe that the algorithm has nearly zero mean lateral error distribution and a low standard deviation $\sigma$. This distribution profile error is due to the inclusion of lane-boundary constraints. The cumulative distribution $3 \sigma$ boundary of the lateral positioning error for $L B-A S G P$ algorithm is greatly reduced meaning that the positioning method has an accuracy of better than one meter in simulation and 2.5 meters in field experiments, most of the time. The $3 \sigma$ is reduced by at least one factor of 4 when using the $L B-A S G P$ algorithm. From center subplots of Fig. 5 and Table IV, one can observe that algorithm has nearly zero mean longitudinal spread error distribution and a high standard deviation $\sigma$. As expected, this distribution profile is similar to the one obtained without the laneboundary constraints, since the lane-boundary sensors only provide additional geometric diversity for the axis orthogonal to the road lane. The cumulative distribution $3 \sigma$ boundary of the longitudinal positioning error for $L B-A S G P$ algorithm maintains a very high value as expected.

The bottom subplots of Fig. 5 and Table IV present the 2D Euclidean error distribution. For both simulations and field experiments, the mean and standard deviation $\sigma$ error of the $L B$-ASGP algorithm are reduced, this being more evident on the field experiment, therefore validating the simulations.

Figure 6(a) shows the trajectory followed by all vehicles and position estimation for all presented algorithms in simulation. The subplots represent the zoomed trajectories for vehicles 1 , 2, 4 and 6 respectively bottom-left, bottom-right, top-left and top-right zoom subplots. As mentioned earlier, this satellite constellation configuration generates errors along both $X$ and $Y$ local coordinates axes, with the major error axis along the negative direction of the local coordinates $X$-axis (see position estimation of vehicles 2 and 4 using $S G P$ in Fig. 6(a)). Therefore, the improvement of position estimation by using lane-boundary sensors is more noticeable for vehicles travelling along the local coordinates $Y$-axis and with the laneboundary sensors measurements along the local coordinates $X$-axis, as with vehicles 2 and 4 . The position estimation improvement of $L B-A S G P$ algorithm for vehicles 2 and 4 is shown in subplots bottom-right and top-left respectively, where 

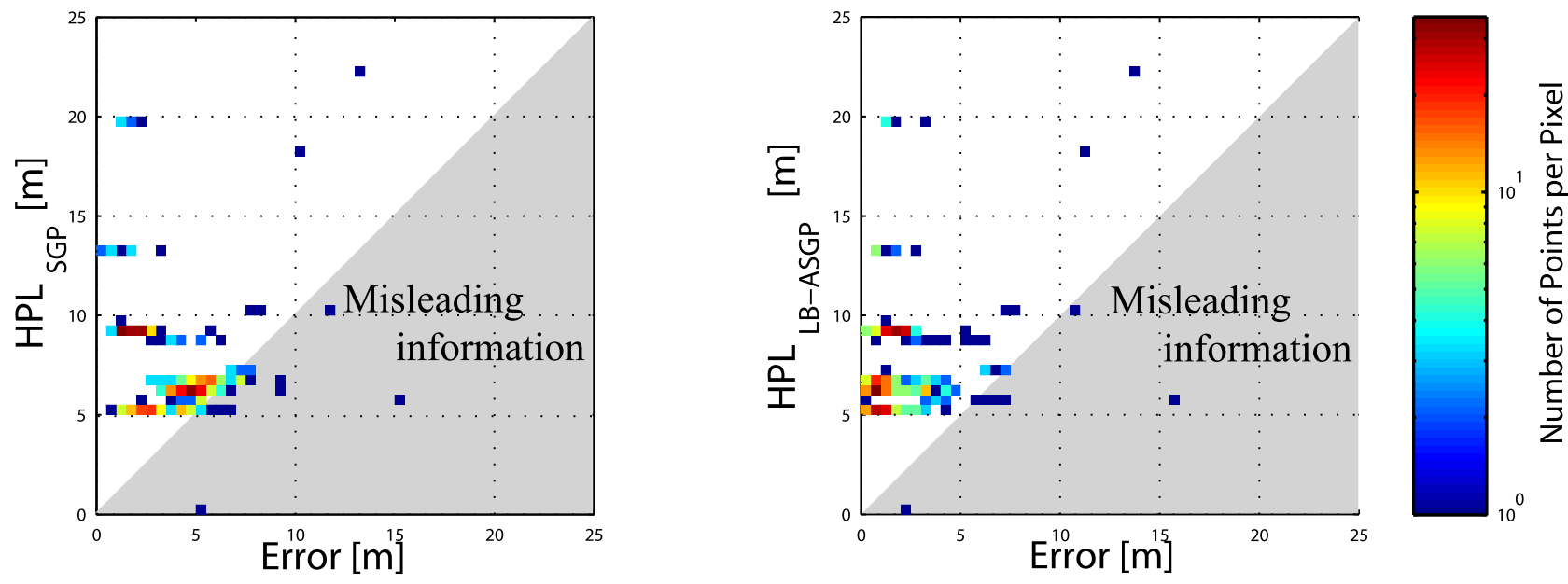

Fig. 7. Field experiments - simplified HPL Stanford diagram for the two set-membership methods.

the position estimate is shifted right towards the real position. The position estimation improvement for vehicles 1 and 6 is less evident since these vehicles travel along the local $\mathrm{x}$-coordinate and the error along the local $\mathrm{y}$-coordinate is medium positive (see position estimation of vehicles 1 and 6 using $S G P$ in Fig. 6(a)). The estimates of vehicles 1 and 6 are shifted down towards the real position, this correction can be observed on the bottom-left and top-right subplots respectively. This correction reflects a medium improvement of $L B-A S G P$ algorithm on the position estimate.

Figure 6(b) shows the trajectory followed by all vehicles and position estimates for all presented algorithms in field experiments. By analysing the subplots of Fig. 6(b), it is possible to observe that the satellite constellation configuration, during the field experiments, generates an error with the major axis along the north-east direction (i.e towards the top-right corner of Fig. 6(b)). This bias is successfully mitigated using the $L B$-ASGP algorithm.

One can observe that the mean, standard deviation, maximum and minimum errors in positioning are similar for both $L B$-AIGP and $L B-A S G P$ algorithms, being slightly higher for the $L B$-ASGP algorithm. The $L B$-AIGP and $L B-A S G P$ algorithms have significantly less mean absolute lateral error than IGP and SGP, with an error reduction of at least $90 \%$.

\section{E. Integrity Analysis}

If the solution set of $S G P$ or $L B-A S G P$ is an empty set, a fault is detected. The method then directly signals an integrity failure and the epoch is discarded, meaning that there is no position estimation available (see Fig. 2).

Horizontal Protection Level (HPL) and Vertical Protection Level (VPL) are often used in the context of aviation integrity analysis [26]. Regarding the context of intelligent vehicles only HPL is of interest [25]. A common representation of the histogram of positioning solutions in terms of actual error and protection level, is through the use of Stanford diagrams.

Figure 7 is a simplified HPL Stanford diagrams. We have implemented a classical Receiver Autonomous Integrity Monitoring (RAIM) algorithm. The HPL computation was tuned with a probability of missed detection equal to 0.001 and a false alarm rate of $3.33333 \times 10^{-7}$. A misleading information event occurs when the position error is not bounded by the HPL.

Figure 7 shows the integrity performance of both $S G P$ and $L B$-ASGP punctual estimation, achieved in field experiments during time periods analyzed. The RAIM algorithm was implemented using the estimates provided by each algorithm (SGP or $L B-A S G P$ ). For each algorithm, the navigation solution of all the vehicles has been merged in the same plot in order to show the total domain integrity per algorithm.

A significant improvement is achieved by using $L B-A S G P$ since the system is under normal operation (i.e. without misleading information events) $93 \%$ of the time. The SGP algorithm is $88 \%$ of the time under normal operation. For some samples, the error exceeds the HPL and this situation is six times higher for $S G P$ than for $L B-A S G P$. We can therefore conclude that the lane-boundary measurements also improve the integrity of the computation.

\section{CONCLUSion AND Future Work}

This paper has presented an enhancement to a previous algorithm (SGP) [15], based on constraints propagation on real intervals. This new positioning algorithm (LB-ASGP) uses a lane-boundary sensor capable of measuring the distance from the vehicle to the lane, therefore improving the performance of a standalone GNSS algorithm. The two set-inversion based algorithms (i.e. $S G P$ and $L B-A S G P$ ) are very reliable, i.e. they are insensitive to local minimum convergence issues. They also have the advantage of not losing any solution in the computation process, and the risk taken of failing to include the solution in the set is only dependant on the risk taken on setting the bounds on the measurements.

Using the same software implementation in both simulation and field experiments, the results revealed improvements in both magnitude of errors and confidence domains. Positioning using non-augmented (IGP and $S G P$ ) algorithms has similar results. This fact is also observable with the augmented (LB-AIGP and $L B-A S G P$ ) algorithms where both algorithms 
have similar results. In terms of accuracy, the lane-aided algorithm demonstrates a significantly better performance than the algorithm that relies on GNSS only. In particular, the main part of the cross-track errors associated to the GNSS positioning as concerns the algorithm $L B-A S G P$ is significantly reduced by the data fusion of the geo-referenced lane boundary measurements. We have noticed that integrity is also improved thanks to the map measurements.

\section{REFERENCES}

[1] A. Amditis, M. Da Lio, and R. Goudy, "Guest editorial special section on ITS and road safety," IEEE Trans. Intell. Transp. Syst., vol. 11, no. 3, p. 524, Sep. 2010

[2] M. Á. Sotelo, J. W. C. van Lint, U. Nunes, L. B. Vlacic, and M. Chowdhury, "Introduction to the special issue on emergent cooperative technologies in intelligent transportation systems," IEEE Trans. Intell. Transp. Syst., vol. 13, no. 1, pp. 1-5, Mar. 2012.

[3] L. C. Bento, P. Bonnifait, and U. J. Nunes, "Cooperative GNSS positioning aided by road-features measurements," Transp. Res. C, Emerg. Technol., vol. 79, pp. 42-57, Jul. 2017.

[4] N. M. Drawil and O. Basir, "Intervehicle-communication-assisted localization," IEEE Trans. Intell. Transp. Syst., vol. 11, no. 3, pp. 678-691, Sep. 2010.

[5] W. Li and H. Leung, "Simultaneous registration and fusion of multiple dissimilar sensors for cooperative driving," IEEE Trans. Intell. Transp. Syst., vol. 5, no. 2, pp. 84-98, Jun. 2004.

[6] W.-B. Zhang, R. E. Parsons, T. West, "An intelligent roadway reference system for vehicle lateral guidance/control," in Proc. Amer. Control Conf., May 1990, pp. 281-286.

[7] H. Li, F. Nashashibi, and G. Toulminet, "Localization for intelligent vehicle by fusing mono-camera, low-cost GPS and map data," in Proc. IEEE Int. Conf. Intell. Transp. Syst. (ITSC), Sep. 2010, pp. 1657-1662.

[8] Z. Tao and P. Bonnifait, "Sequential data fusion of GNSS pseudoranges and Dopplers with map-based vision systems," IEEE Trans. Intell. Vehicles, vol. 1, no. 3, pp. 254-265, Sep. 2016.

[9] S. Kammel and B. Pitzer, "Lidar-based lane marker detection and mapping," in Proc. IEEE Intell. Vehicles Symp., Eindhoven, The Netherlands, Jun. 2008, pp. 1137-1142.

[10] S. Dominguez, B. Khomutenko, G. Garcia, and P. Martinet, "An optimization technique for positioning multiple maps for self-driving car's autonomous navigation," in Proc. IEEE Int. Conf. Intell. Transp. Syst., Las Palmas, Spain, Sep. 2015, pp. 2694-2699.

[11] J. Rife and X. Xiao, "Estimation of spatially correlated errors in vehicular collaborative navigation with shared GNSS and road-boundary measurements," in Proc. Int. Tech. Meeting Satellite Division Inst. Navigat. (ION GNSS), Portland, OR, USA, Sep. 2010, pp. 1667-1677.

[12] J. Rife, "Collaborative vision-integrated pseudorange error removal: Team-estimated differential GNSS corrections with no stationary reference receiver," IEEE Trans. Intell. Transp. Syst., vol. 13, no. 1, pp. 15-24, Mar. 2012.

[13] S. Miura, S. Hisaka, and S. Kamijo, "GPS multipath detection and rectification using 3D maps," in Proc. IEEE Int. Conf. Intell. Transp. Syst., Hague, Netherlands, Oct. 2013, pp. 1528-1534.

[14] V. Drevelle and P. Bonnifait, "A set-membership approach for high integrity height-aided satellite positioning," GPS Solutions, vol. 15, no. 4, pp. 357-368, 2011.

[15] V. Drevelle and P. Bonnifait, "Reliable positioning domain computation for urban navigation," IEEE Intell. Transp. Syst. Mag., vol. 5, no. 3, pp. 21-29, Fall 2013.

[16] V. Drevelle and P. Bonnifait, "Localization confidence domains via set inversion on short-term trajectory," IEEE Trans. Robot., vol. 29, no. 5, pp. 1244-1256, Oct. 2013.

[17] M. Wörner, F. Schuster, F. Dölitzscher, C. G. Keller, M. Haueis, and K. Dietmayer, "Integrity for autonomous driving: A survey," in Proc. IEEE Position, Location Navigat. Symp. (PLANS), Savannah, GA, USA, Apr. 2016, pp. 666-671.

[18] S. Tornil-Sin, V. Puig, and T. Escobet, "Set computations with subpavings in MATLAB: The SCS toolbox," in Proc. IEEE Int. Symp. Comput.-Aided Control Syst. Design (CACSD), Sep. 2010, pp. 1403-1408.

[19] A. Gning and P. Bonnifait, "Constraints propagation techniques on intervals for a guaranteed localization using redundant data," J. Autom., vol. 42, no. 7, pp. 1167-1175, Jul. 2006.
[20] E. D. Kaplan, Understanding GPS: Principles and Applications. Boston, MA, USA: Artech House, 1996.

[21] A. K. Tetewsky and A. Soltz, "GPS MATLAB toolbox review," GPS World, vol. 9, no. 10, pp. 50-57, 1998

[22] L. Jaulin, M. Kieffer, O. Didrit, and E. Walter, Applied Interval Analysis. New York, NY, USA: Springer-Verlag, 2001.

[23] L. Jaulin and E. Walter, "Guaranteed nonlinear estimation and robust stability analysis via set inversion," in Proc. Control Conf. ECC, Groningen, The Netherlands, 1993, pp. 818-821.

[24] I. Gat, M. Benady, and A. Shashua, "A monocular vision advance warning system for the automotive aftermarket," SAE Tech. Paper 200501-1470, 2005.

[25] J. Santa, B. Ubeda, R. Toledo, and A. F. G. Skarmeta, "Monitoring the position integrity in road transport localization based services," in Proc. IEEE 64th Veh. Technol. Conf. (VTC), Sep. 2006, pp. 1-5.

[26] M. Tossaint et al., "The Stanford-ESA integrity diagram: A new tool for the user domain SBAS integrity assessment," J. Inst. Navigat., vol. 54, no. 2, pp. 153-162, 2007.

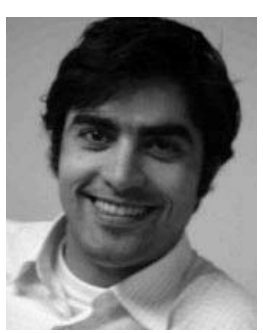

Luís Conde Bento received the Ph.D. degree in electrical engineering from the University of Coimbra, Portugal, in 2016. Since 2002, he has been a Researcher with the Institute for Systems and Robotics, University of Coimbra. He is currently a Professor with the Electrical Engineering Department, School of Technology and Management, Polytechnic Institute of Leiria, Portugal. He has also been enrolled in several funded research and development projects acting either as a co-manager or a supervisor, as well as hardware and middleware developer for embedded systems. His research interests include automatic control, GNSS positioning systems, autonomous vehicles, and traffic management.

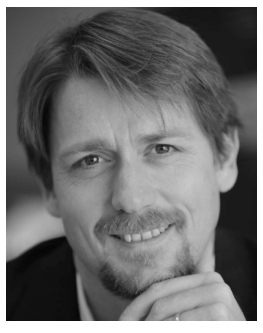

Philippe Bonnifait received the Ph.D. degree in automatic control and computer science from the École Centrale de Nantes in 1997. Since 1998, he has been with the Heudiasyc UMR 7253, a joint research unit between the Université de Technologie de Compiègne (UTC) and CNRS. He is currently a Professor with the Computer Science and Engineering Department, UTC, France. His research interests include intelligent vehicles, and high-integrity positioning and map-matching for autonomous navigation in structured outdoor environments.

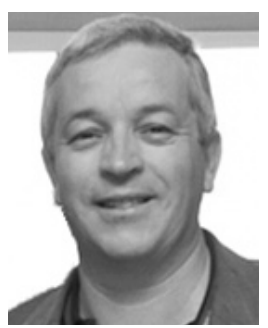

Urbano J. Nunes received the Ph.D. degree in electrical engineering from the University of Coimbra, Portugal, in 1995. He is currently a Full Professor with the Electrical and Computer Engineering Department, University of Coimbra (UC). He is also a Researcher with the Institute for Systems and Robotics, UC, where has been a Coordinator of the Automation and Robotics for Human Life Group. He has been involved with/responsible for several funded projects at both national and international levels in the areas of mobile robotics, intelligent vehicles, and intelligent transportation systems (ITSs). He was a member of the Board of Governors of the ITS Society from 2009 to 2011, the Vice President for Technical Activities of the IEEE ITS Society from 2011 to 2012, and a Co-chair of the Technical Committee on Autonomous Ground Vehicles and ITS from 2006 to 2011 and the IEEE Robotics and Automation Society. $\mathrm{He}$ has been active in the organization of conferences, such as the Program Chair of the 2006 IEEE Intelligent Transportation Systems Conference, the General Chair of the 2010 IEEE Intelligent Transportation Systems Conference, the General Chair of the 2012 IEEE/RSJ Intelligent Robots and Systems, and the General Chair of the 2017 IEEE International Symposium on Robot and Human Interactive Communication. $\mathrm{He}$ is an Associate Editor of the IEEE TRANSACTIONS ON INTELLIGENT VehicLES. 